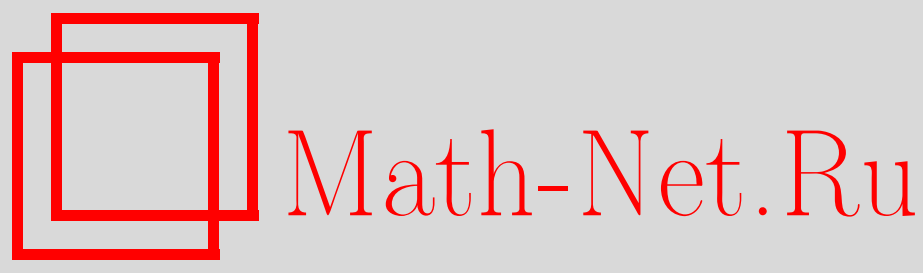

В. В. Напалков, А. А. Нуятов, Многоточечная задача Валле Пуссена для операторов свертки с узлами, заданными в угле, ТMФ, 2014, том 180, номер 2, 264-271

DOI: https://doi.org/10.4213/tmf8654

Использование Общероссийского математического портала Math-Net.Ru подразумевает, что вы прочитали и согласны с пользовательским соглашением http://www.mathnet.ru/rus/agreement

Параметры загрузки:

IP: 3.95.254.165

26 апреля 2023 г., 13:48:27

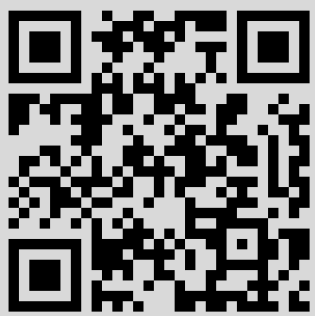




\title{
ФИЗИКА
}

Том 180, № 2

август, 2014

\author{
(C) 2014 г. \\ В. В. Напалков* , А. А. Нуятов ${ }^{\dagger}$
}

\section{МНОГОТОЧЕЧНАЯ ЗАДАЧА ВАЛЛЕ ПУССЕНА ДЛЯ ОПЕРАТОРОВ СВЕРТКИ С УЗЛАМИ, ЗАДАННЫМИ В УГЛЕ}

\begin{abstract}
Доказана разрешимость многоточечной задачи Валле Пуссена (задачи интерполяции) в ядре оператора свертки, когда нули характеристической функции и узловые точки, являющиеся нулями целой функции, содержатся внутри некоторых углов.
\end{abstract}

Ключевые слова: оператор свертки, многоточечная задача Валле Пуссена, интерполяция, пары Фишера.

DOI: $10.4231 / \operatorname{tmf} 8654$

\section{1. ВВЕДЕНИЕ}

Пусть $H(\mathbb{C})$ - пространство целых функций с топологией равномерной сходимости на компактах, $H^{*}(\mathbb{C})$ - пространство, сопряженное к $H(\mathbb{C}), P_{\mathbb{C}}-$ пространство целых функций экспоненциального роста. Сопоставим функции $\varphi \in P_{\mathbb{C}}$ функционал $F \in H^{*}(\mathbb{C})$ такой, что $\widehat{F}(z)=\varphi(z)$, где $\widehat{F}(z)=\left\langle F_{\lambda}, e^{\lambda z}\right\rangle-$ преобразование Лапласа функционала $F$. Оператор свертки в $H(\mathbb{C})$ запишем в виде

$$
M_{\varphi}[f](z)=\left(F_{t}, f(z+t)\right), \quad f \in H(\mathbb{C}) .
$$

Обозначим через $\operatorname{Ker} M_{\varphi}$ ядро оператора свертки $M_{\varphi}$,

$$
\operatorname{Ker} M_{\varphi}=\left\{f \in H(\mathbb{C}): M_{\varphi}[f]=0\right\} .
$$

Многоточечную задачу Валле Пуссена в $\operatorname{Ker} M_{\varphi}$ с узлами $\mu_{j}, j=1,2, \ldots$, являющимися нулями функции $\psi \in H(\mathbb{C})$, поставим следующим образом: существует ли для произвольной последовательности комплексных чисел $a_{j}, j=1,2, \ldots$, функция $y \in \operatorname{Ker} M_{\varphi}$ такая, что $y\left(\mu_{j}\right)=a_{j}$ для всех $j=1,2, \ldots$ ? В работе [1] эта задача решена для случая, когда узлы лежат на вещественной оси. В настоящей статье мы существенно обобщим постановку задачи, а именно будем считать, что узлы лежат в некотором угле, т. е. могут быть комплексными.

* Институт математики с вычислительным центром Уфимского научного центра РАН, Уфа, Россия. Е-mail: shaig@anrb.ru

${ }^{\dagger}$ Нижегородский государственный университет им. Н. И. Лобачевского, Нижний Новгород, Россия. E-mail: nuyatov1aa@rambler.ru 


\section{2. ПРЕДВАРИТЕЛЬНЫЕ РЕЗУЛЬТАТЫ}

Разрешимость многоточечной задачи Валле Пуссена тесно связана с таким понятием, как пары Фишера.

ОПРЕДЕЛЕниЕ 1. Пара функций $(\varphi(z), \psi(z))$ называется парой Фишера, если пространство $H(\mathbb{C})$ можно представить в виде

$$
H(\mathbb{C})=\operatorname{Ker} M_{\varphi}+(\psi),
$$

где $(\psi)=\{\psi(\lambda) \cdot R(\lambda): R(\lambda) \in H(\mathbb{C})\}$ - главный идеал в пространстве $H(\mathbb{C})$. Равенство (1) называется представление Фишера.

В работе [2] доказана

Теорема 1. Следующие утверждения эквивалентны:

1) задача Валле Пуссена для оператора $M_{\varphi}$ с данными на множестве нулей функи,ии $\psi(z) \in H(\mathbb{C})$ разрешима;

2) имеет место представление Фишера.

Выясним, при каких условиях существует представление Фишера, для чего введем ряд вспомогательных понятий.

Топология $\tau_{\mathbb{C}}$ пространства $P_{\mathbb{C}}$ определяется как индуктивный предел нормированных весовых пространств

$$
B_{n}=\left\{\varphi(\lambda) \in P_{\mathbb{C}}:\|\varphi\|_{n}=\sup _{\lambda \in \mathbb{C}}|\varphi(\lambda)| e^{-n|\lambda|}<\infty\right\}, \quad n=1,2, \ldots
$$

Пусть $S \subset \mathbb{C}$ - множество единственности в $P_{\mathbb{C}}$. Тогда в $P_{\mathbb{C}}$ можно ввести топологию $\tau_{S}$ индуктивного предела пространств

$$
B_{n, S}=\left\{\varphi(\lambda) \in P_{\mathbb{C}}:\|\varphi\|_{n, S}=\sup _{\lambda \in S}|\varphi(\lambda)| e^{-n|\lambda|}<\infty\right\}, \quad n=1,2, \ldots .
$$

ОПРеДЕЛЕнИЕ 2. Множество $S$ называется секвенциально достаточным в пространстве $P_{\mathbb{C}}$, если сходимость последовательности $r_{m}(z) \rightarrow 0$ при $m \rightarrow \infty$ в топологии $\tau_{\mathbb{C}}$ эквивалентна сходимости последовательности $r_{m}(z) \rightarrow 0$ при $m \rightarrow \infty$ в топологии $\tau_{S}$.

Функция $\psi(z) \in H(\mathbb{C})$ порождает (см. работу [3]) в пространстве $P_{\mathbb{C}}$ линейный и непрерывный оператор $M_{\psi}: P_{\mathbb{C}} \rightarrow P_{\mathbb{C}}$, действующий по правилу

$$
M_{\psi}[f](z)=\frac{1}{2 \pi i} \int_{C} e^{z \xi} \psi(\xi) \gamma(\xi) d \xi,
$$

где $\gamma(\xi)$ - функция, ассоциированная по Борелю с $f(z), C$ - замкнутый контур, охватывающий все особые точки функции $\gamma(\xi)$.

Обозначим через $N_{\varphi}$ множество нулей функции $\varphi \in P_{\mathbb{C}}$. В работе [1] доказана

Tеорема 2. Пусть $\varphi \in P_{\mathbb{C}}, \psi \in H(\mathbb{C})$ и множество $N_{\varphi}$ является секвенииально достаточным в $\operatorname{Ker} M_{\psi}$. Тогда имеет место представление Фишера

$$
H(\mathbb{C})=\operatorname{Ker} M_{\varphi}+(\psi) .
$$




\section{3. ОСНОВНОЙ РЕЗУЛЬТАТ}

Пусть $N_{\varphi}=\left\{\lambda_{k}\right\}_{k=1}^{+\infty}-$ бесконечное множество нулей функции $\varphi \in P_{\mathbb{C}}$, каждый нуль повторяется столько раз, какова его кратность; $N_{\psi}=\left\{\mu_{k}\right\}_{k=1}^{+\infty}$-бесконечное множество простых нулей функции $\psi \in H(\mathbb{C})$.

Теорема 3. Предположим, что для некоторого фиксированного $\alpha \in[0,+\infty)$ число $\beta \in[0,+\infty)$ таково, что $\alpha \beta<1$, и при этом выполнены следующие условия:

1) имеет место включение $N_{\varphi} \subset D_{\alpha}=\{z \in \mathbb{C}:|\operatorname{Im} z| \leqslant \alpha \operatorname{Re} z\}$, и существует подпоследовательность $\lambda_{k_{s}}$ такая, что

$$
\operatorname{Re} \lambda_{k_{s}}<\operatorname{Re} \lambda_{k_{s+1}}, \quad s=1,2, \ldots
$$

2) имеет место включение $N_{\psi} \subset D_{\beta}=\{z \in \mathbb{C}:|\operatorname{Im} z| \leqslant \beta \operatorname{Re} z\}$, причем

$$
\operatorname{Re} \mu_{k}<\frac{1-\alpha \beta}{1+\alpha \beta} \operatorname{Re} \mu_{k+1}, \quad k=1,2, \ldots
$$

Тогда $N_{\varphi}$ является секвенциально достаточным в $\operatorname{Ker} M_{\psi}$.

ДокАЗАтельство. Согласно результату работы [3] пространство Ker $M_{\psi}$ состоит из квазиполиномов с показателями из множества $N_{\psi}$, другими словами, любой элемент $r(z) \in \operatorname{Ker} M_{\psi}$ можно записать в виде

$$
r(z)=\sum_{j=1}^{N} C_{j} e^{\mu_{j} z} .
$$

Пусть последовательность

$$
r_{m}(z)=\sum_{j=1}^{N(m)} C_{j}(m) e^{\mu_{j} z}, \quad m=1,2, \ldots
$$

стремится к нулю в топологии $\tau_{N_{\varphi}}$. По известным свойствам рассматриваемых топологий [4] и с учетом дискретности множества $N_{\varphi}$ условие сходимости к нулю в топологии $\tau_{N_{\varphi}}$ можно записать в следующем виде: для некоторых постоянных $\sigma, M>0$ выполняются соотношения

$$
\left|r_{m}\left(\lambda_{k}\right)\right| \leqslant M e^{\sigma\left|\lambda_{k}\right|}, \quad m=1,2, \ldots, \quad k=1,2, \ldots,
$$

и для любого $k=1,2, \ldots$ последовательность сходится равномерно на компактных подмножествах $N_{\varphi}$, т. е.

$$
\left|r_{m}\left(\lambda_{k}\right)\right| \rightarrow 0, \quad m \rightarrow \infty
$$

Требуется показать, что $r_{m} \rightarrow 0$ в топологии $\tau_{\mathbb{C}}$.

1. Докажем, что при выполнении условия (5) имеет место оценка

$$
\operatorname{Re} \mu_{j} \leqslant \frac{(1+\alpha) \sigma}{1-\alpha \beta}, \quad j=1, \ldots, N
$$


Проведем доказательство от противного. Пусть для фиксированного $m$ все значения $\mu_{j}, j=1, \ldots, N$, упорядочены по возрастанию реальных частей. Предположим, что

$$
\operatorname{Re} \mu_{N}>\frac{(1+\alpha) \sigma}{1-\alpha \beta}
$$

Рассмотрим отношение

$$
\frac{\left|e^{\mu_{j} \lambda_{k}}\right|}{\left|e^{\mu_{N} \lambda_{k}}\right|} \leqslant \frac{e^{(1+\alpha \beta) \operatorname{Re} \mu_{j} \operatorname{Re} \lambda_{k}}}{e^{(1-\alpha \beta) \operatorname{Re} \mu_{N} \operatorname{Re} \lambda_{k}}}=e^{(1+\alpha \beta) \operatorname{Re} \mu_{j} \operatorname{Re} \lambda_{k}-(1-\alpha \beta) \operatorname{Re} \mu_{N} \operatorname{Re} \lambda_{k}} .
$$

Для $j=1, \ldots, N-1$ в силу условия (3) показатель экспоненты отрицателен, поэтому отношение модулей стремится к нулю при $\operatorname{Re} \lambda_{k} \rightarrow+\infty$. Из неравенства (9) следует, что

$$
\left|\sum_{j=1}^{N} C_{j}(m) e^{\left(\mu_{j}-\mu_{N}\right) \lambda_{k}}\right| \rightarrow\left|C_{N}(m)\right|, \quad \operatorname{Re} \lambda_{k} \rightarrow+\infty,
$$

поэтому рост квазиполинома определяется величиной $\mu_{N}$. С другой стороны, рост квазиполинома $r_{m}\left(\lambda_{k}\right)$ задается с помощью (5). Записав оценки сверху и снизу в (5), получаем цепочку неравенств

$$
\left|\sum_{j=1}^{N} C_{j}(m) e^{\left(\mu_{j}-\mu_{N}\right) \lambda_{k}}\right| e^{(1-\alpha \beta) \operatorname{Re} \mu_{N} \operatorname{Re} \lambda_{k}} \leqslant\left|r_{m}\left(\lambda_{k}\right)\right| \leqslant M e^{\sigma\left|\lambda_{k}\right|} \leqslant M e^{\sigma(1+\alpha) \operatorname{Re} \lambda_{k}} .
$$

Рассмотрим крайние элементы неравенства и перепишем данное неравенство как

$$
\frac{\left|\sum_{j=1}^{N} C_{j}(m) e^{\left(\mu_{j}-\mu_{N}\right) \lambda_{k}}\right| e^{(1-\alpha \beta) \operatorname{Re} \mu_{N} \operatorname{Re} \lambda_{k}}}{e^{\sigma(1+\alpha) \operatorname{Re} \lambda_{k}}} \leqslant M
$$

или, что эквивалентно, как

$$
\left|\sum_{j=1}^{N} C_{j}(m) e^{\left(\mu_{j}-\mu_{N}\right) \lambda_{k}}\right| e^{\left((1-\alpha \beta) \operatorname{Re} \mu_{N}-\sigma(1+\alpha)\right) \operatorname{Re} \lambda_{k}} \leqslant M
$$

Из неравенства (8) следует, что левая часть неравенства (10) стремится к $+\infty$, когда $\operatorname{Re} \lambda_{k} \rightarrow+\infty$. Получаем противоречие с тем, что левая часть должна быть ограничена.

Таким образом, $\operatorname{Re} \mu_{N} \leqslant(1+\alpha) \sigma /(1-\alpha \beta)$. Мы показали, что в представлении (4) участвуют лишь $e^{\mu_{j} z}$ с показателем $\mu_{j}$, удовлетворяющим оценке (7). Поскольку $\mu_{j}$ - нули целой функции, показателей, участвующих в представлении (4), конечное число.

2. Докажем далее, что если последовательность квазиполиномов $r_{m}(z)$ стремится к нулю в топологии $\tau_{N_{\varphi}}$, то коэффициенты $C_{j}(m)$ ограничены, т. е. найдется константа $C$ такая, что $\left|C_{j}(m)\right| \leqslant C$ для всех $j=1, \ldots, N$ и $m=1,2, \ldots$ Чтобы не было громоздких обозначений, в дальнейшем под $\lambda_{\tilde{k}}, \tilde{k}=1,2, \ldots$, будем понимать некоторую подпоследовательность последовательности $\left\{\lambda_{i}\right\}_{i=1}^{+\infty}$. Пусть для некоторого $N=N(m)$

$$
r_{m}\left(\lambda_{\tilde{k}}\right)=\sum_{j=1}^{N} C_{j}(m) e^{\mu_{j} \lambda_{\tilde{k}}}, \quad m=1,2, \ldots
$$


Мы воспользуемся простым замечанием, что если набор из $N$ нулей $\lambda_{\tilde{k}_{p}}$ выбрать так, что определитель матрицы

$$
A=\left(e^{\mu_{j} \lambda_{\tilde{k}_{p}}}\right), \quad j, p=1,2, \ldots, N
$$

отличен от нуля, то коэффициенты $C_{j}(m)$ являются решением системы линейных уравнений

$$
\sum_{j=1}^{N} C_{j} e^{\mu_{j} \lambda_{\tilde{k}_{p}}}=r_{m}\left(\lambda_{\tilde{k}_{p}}\right), \quad p=1,2, \ldots, N
$$

Докажем по индукции по параметру $t=1,2, \ldots$, что набор нулей можно выбрать так, что модули определителей матриц

$$
A(t)=\left(e^{\mu_{j} \lambda_{\tilde{k}_{p}}}\right), \quad j, p=1,2, \ldots, t
$$

будут больше единицы. Вначале рассмотрим $t=1$ :

$$
|\operatorname{det} A(1)|=e^{\operatorname{Re}\left(\mu_{1} \lambda_{\tilde{k}_{1}}\right)} \geqslant e^{(1-\alpha \beta) \operatorname{Re} \mu_{1} \operatorname{Re} \lambda_{\tilde{k}_{1}}} \geqslant 1 .
$$

В качестве $\lambda_{\tilde{k}_{1}}$ можно взять первый элемент последовательности $\lambda_{\tilde{k}}$.

Допустим, что нули $\lambda_{\tilde{k}_{p}}, p=1,2, \ldots, t-1$, выбраны нужным образом, причем $\operatorname{Re} \lambda_{\tilde{k}_{p}}$ возрастает по $p$. Определитель матрицы $A_{t}$ разложим по последней строке:

$$
\operatorname{det} A(t)=\sum_{j=1}^{t}(-1)^{j+t} \operatorname{det} A_{j, t} e^{\mu_{j} \lambda_{\tilde{k}_{t}}}
$$

где $(-1)^{j+t} \operatorname{det} A_{j, t}$ - алгебраическое дополнение элемента $e^{\mu_{j} \lambda_{\tilde{k}_{t}}}$. Отсюда

$$
|\operatorname{det} A(t)| \geqslant\left|\operatorname{det} A_{t, t}\right| e^{\operatorname{Re}\left(\mu_{t} \lambda_{\tilde{k}_{t}}\right)}-\left|\sum_{j=1}^{t-1}(-1)^{j+t} \operatorname{det} A_{j, t} e^{\mu_{j} \lambda_{\tilde{k}_{t}}}\right| .
$$

Оценим сверху $\left|\operatorname{det} A_{j, t}\right|$. Этот определитель есть сумма $(N-1)$ ! слагаемых, при этом каждое слагаемое есть произведение $N-1$ множителей вида $e^{\mu_{j} \lambda_{\tilde{k}_{p}}}, j=1,2, \ldots, t$, $p=1,2, \ldots, t-1$. Учитывая монотонность наборов $\mu_{j}$ и $\lambda_{\tilde{k}_{p}}$, получим

$$
\left|\operatorname{det} A_{j, t}\right| \leqslant(N-1) ! e^{(N-1)(1+\alpha \beta) \operatorname{Re} \mu_{t} \operatorname{Re} \lambda_{\tilde{k}_{t-1}},}
$$

поэтому

$$
\begin{aligned}
& \left|\sum_{j=1}^{t-1}(-1)^{j+t} \operatorname{det} A_{j, t} e^{\mu_{j} \lambda_{\tilde{k}_{t}}}\right| \leqslant \\
& \quad \leqslant(N-1) !(N-1) e^{(N-1)(1+\alpha \beta) \operatorname{Re} \mu_{t} \operatorname{Re} \lambda_{\tilde{k}_{t-1}}+(1+\alpha \beta) \operatorname{Re} \mu_{t-1} \operatorname{Re} \lambda_{\tilde{k}_{t}}} .
\end{aligned}
$$


Алгебраическое дополнение $A_{t, t}$ совпадает с определителем матрицы $A_{t-1}$, и по допущению индукции $\operatorname{det} A_{t-1} \geqslant 1$. Из неравенства (11) теперь имеем

$$
\begin{aligned}
& |\operatorname{det} A(t)| \geqslant \\
& \geqslant e^{(1-\alpha \beta) \operatorname{Re} \lambda_{\tilde{k}_{t}} \operatorname{Re} \mu_{t}}-(N-1) !(N-1) e^{(N-1)(1+\alpha \beta) \operatorname{Re} \mu_{t} \operatorname{Re} \lambda_{\tilde{k}_{t-1}}} e^{(1+\alpha \beta) \operatorname{Re} \mu_{t-1} \operatorname{Re} \lambda_{\tilde{k}_{t}}}= \\
& =e^{(1-\alpha \beta) \operatorname{Re} \mu_{t} \operatorname{Re} \lambda_{\tilde{k}_{t}}\left[1-(N-1) !(N-1) e^{(N-1)(1+\alpha \beta) \operatorname{Re} \mu_{t} \operatorname{Re} \lambda_{\tilde{k}_{t-1}}} \times\right.} \\
& \quad \times e^{\left.(1+\alpha \beta) \operatorname{Re} \mu_{t-1} \operatorname{Re} \lambda_{\tilde{k}_{t}}-(1-\alpha \beta) \operatorname{Re} \mu_{t} \operatorname{Re} \lambda_{\tilde{k}_{t}}\right]} .
\end{aligned}
$$

В правой части последнего равенства первая экспонента в квадратных скобках является фиксированной величиной, а показатель второй экспоненты в силу условия (3) отрицателен. Таким образом, если действительную часть нуля $\lambda_{\tilde{k}_{t}}$ выбрать достаточно большой, то $\Delta=|\operatorname{det} A(t)|$ будет больше единицы.

В построенном определителе в силу условия (2) нули $\lambda_{\tilde{k}_{p}}$ выбраны таким образом, чтобы не было одинаковых строк. Докажем, что в определителе $\Delta$ нет одинаковых столбцов. Возьмем элемент из произвольно выбранной строки и не из последнего столбца, имеем для $i=1,2, \ldots, N-1$

$$
\begin{aligned}
\left|e^{\mu_{i} \lambda_{\tilde{k}_{p}}}\right| & =e^{\operatorname{Re}\left(\mu_{i} \lambda_{\tilde{k}_{p}}\right)} \leqslant e^{(1+\alpha \beta) \operatorname{Re} \mu_{i} \operatorname{Re} \lambda_{\tilde{k}_{p}}}<e^{(1+\alpha \beta) \frac{1-\alpha \beta}{1+\alpha \beta} \operatorname{Re} \mu_{i+1} \operatorname{Re} \lambda_{\tilde{k}_{p}}}= \\
& =e^{(1-\alpha \beta) \operatorname{Re} \mu_{i+1} \operatorname{Re} \lambda_{\tilde{k}_{p}}} \leqslant e^{\operatorname{Re}\left(\mu_{i+1} \lambda_{\tilde{k}_{p}}\right)}=\left|e^{\mu_{i+1} \lambda_{\tilde{k}_{p}}}\right|
\end{aligned}
$$

Таким образом, для произвольной строки получаем цепочку неравенств

$$
\left|e^{\mu_{1} \lambda_{\tilde{k}_{p}}}\right|<\left|e^{\mu_{2} \lambda_{\tilde{k}_{p}}}\right|<\cdots<\left|e^{\mu_{N} \lambda_{\tilde{k}_{p}}}\right|
$$

По правилу Крамера

$$
C_{j}(m)=\frac{\Delta_{j}}{\Delta}
$$

где $\Delta_{j}$ - определитель матрицы, полученной из $A_{N}$ заменой $j$-го столбца столбцом свободных членов. Чтобы оценить $\Delta_{j}$, сверху разложим соответствующую матрицу по $j$-му столбцу:

$$
\Delta_{j}=\sum_{p=1}^{N}(-1)^{j+p} \operatorname{det} A_{j, p} r_{m}\left(\lambda_{\tilde{k}_{p}}\right)
$$

Так же, как соотношение (12), получим оценку

$$
\left|\operatorname{det} A_{j, p}\right| \leqslant(N-1) ! e^{(N-1)(1+\alpha \beta) \operatorname{Re} \mu_{N} \operatorname{Re} \lambda_{\tilde{k}_{N}},}
$$

а по условию (5)

$$
\left|r_{m}\left(\lambda_{\tilde{k}_{p}}\right)\right| \leqslant M e^{(1+\alpha) \sigma \operatorname{Re} \lambda_{\tilde{k}_{N}}}
$$


Таким образом,

$$
\begin{aligned}
\left|\Delta_{j}\right| & \leqslant N ! M e^{(N-1)(1+\alpha \beta) \frac{\sigma(1+\alpha)}{1-\alpha \beta} \operatorname{Re} \lambda_{\tilde{k}_{N}}+\sigma(1+\alpha) \operatorname{Re} \lambda_{\tilde{k}_{N}}}= \\
& =N ! M e^{\left((N-1) \frac{1+\alpha \beta}{1-\alpha \beta}+1\right) \sigma(1+\alpha) \operatorname{Re} \lambda_{\tilde{k}_{N}}}
\end{aligned}
$$

и

$$
\left|C_{j}(m)\right| \leqslant C:=N ! M e^{\left((N-1) \frac{1+\alpha \beta}{1-\alpha \beta}+1\right) \sigma(1+\alpha) \operatorname{Re} \lambda_{\tilde{k}_{N}}} .
$$

3. Докажем, что $N_{\varphi}$ является множеством единственности в $\operatorname{Ker} M_{\psi}$. Для этого надо показать, что из равенства $r_{m}\left(\lambda_{\tilde{k}_{p}}\right)=0$ следует тождество $r_{m}(z) \equiv 0$. Рассмотрим систему

$$
\sum_{j=1}^{N} C_{j}(m) e^{\mu_{j} \lambda_{\tilde{k}_{p}}}=0 .
$$

По доказанному в п. 2 модуль определителя матрицы этой системы будет больше единицы. Выражая коэффициенты $C_{j}(m), j=1,2, \ldots, N$, по правилу Крамера (см. формулу (13)) и заменяя в определителе $\Delta_{j} j$-й столбец столбцом свободных членов, состоящим из нулей, получим, что $C_{j}(m)=0$, а это означает, что $r_{m}(z) \equiv 0$.

4. Докажем, что

$$
\lim _{m \rightarrow \infty} C_{j}(m)=0, \quad j=1,2, \ldots, N .
$$

Из рассуждений предыдущего пункта следует, что для $\left|C_{j}(m)\right|$ в силу соотношения (6)

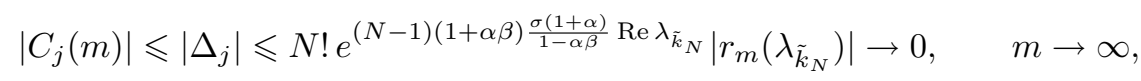

для любых $N=1,2, \ldots$, таким образом,

$$
\lim _{m \rightarrow \infty} C_{j}(m)=0, \quad j=1,2, \ldots, N
$$

5. Закончим доказательство секвенциальной достаточности множества $N_{\varphi}$. Ранее в п. 1, 2 мы доказали, что

$$
\operatorname{Re} \mu_{j} \leqslant \frac{\sigma(1+\alpha)}{1-\alpha \beta}, \quad\left|C_{j}(m)\right| \leqslant C .
$$

Следовательно,

$$
\left|r_{m}(z)\right| \leqslant N\left|C_{j}(m)\right| e^{\operatorname{Re}\left(\mu_{j} \cdot z\right)} \leqslant N C e^{\frac{\sigma(1+\alpha)}{1-\alpha \beta}(1+\beta)|z|}, \quad z \in \mathbb{C} .
$$

В п. 4 мы доказали, что $C_{j}(m) \rightarrow 0$ при любом $j$, следовательно, $\max _{j}\left|C_{j}(m)\right| \rightarrow 0$. Поэтому для любого компакта $K_{\mathbb{C}}$ при $z \in K_{\mathbb{C}}$ мы имеем

$$
\left|r_{m}(z)\right| \leqslant N \cdot \max _{j=1,2, \ldots, N}\left|C_{j}(m)\right| e^{\frac{\sigma(1+\alpha)}{1-\alpha \beta}(1+\beta) \max _{z \in K_{\mathbb{C}}}|z|} \rightarrow 0
$$

Отсюда вытекает, что $r_{m}(z) \rightarrow 0$ в топологии $\tau_{\mathbb{C}}$. 


\section{Список литературы}

[1] В. В. Напалков, А. А. Нуятов, Матем. сб., 203:2 (2012), 77-86.

[2] В. В. Напалков, С. В. Попёнов, Докл. РАН, 381:2 (2001), 164-166.

[3] H. Muggli, Comment. Math. Helv., 11:1 (1938), 151-179.

[4] Ж. Себаштьян-и-Силва, "О некоторых классах локально-выпуклых пространств, важных в приложениях", Математика. Периодический сборник переводов иностранных статей, т. 1, вып. 1, ред. А. О. Гельфонд, ИЛ, М., 1957, 60-77.

Поступила в редакцию 13.02.2014 